\title{
A Database of Electrocardiogram Signals acquired in different Magnetic Resonance Imaging Scanners
}

\author{
Johannes W Krug ${ }^{1}$, Marcus Schmidt ${ }^{2}$, Georg Rose ${ }^{3}$, Michael Friebe ${ }^{1}$ \\ ${ }^{1}$ Chair of Intelligent Catheters; ${ }^{2}$ Chair of Medical Systems Technology; ${ }^{3}$ Chair of Healthcare \\ Telematics and Medical Engineering \\ Department of Medical Engineering, Otto-von-Guericke-University Magdeburg, Germany
}

\begin{abstract}
Magnetic resonance imaging (MRI) is an imaging technique widely used in medical diagnostics as well as in minimally invasive, image guided interventions. During an MRI scan, the patient's electrocardiogram (ECG) can be required for either gating or patient monitoring purposes. However, the hostile environment of an MRI scanner with its various types of magnetic fields (strong static magnetic field, switch gradient magnetic fields in the $\mathrm{Hz}$ or $\mathrm{kHz}$ range as well as high frequency magnetic fields in the $\mathrm{MHz}$ range) causes serve distortions of the acquired ECG signals. These distortions or artifacts hamper the QRS detection and a more detailed ECG-based diagnosis. To study these effects of the MRI environment, we created a database consisting of ECG signals acquired in various MRI scanners. This database was published on Physionet in order to encourage other researchers to enhance the quality of ECG signals during MRI exams.
\end{abstract}

\section{Introduction}

Besides X-ray, computed tomography or ultrasound imaging, magnetic resonance imaging (MRI) is another common medical imaging technique. It is mainly used for medical diagnostics, e. g. for studying the function of the heart or for the detection of cancer but can also provide functional information about the vascular blood flow (angiography), diffusion or spectroscopy. In addition to pure diagnostics, MRI can be used to guide minimally invasive interventions. Such interventions include taking biopsies (e. g. from the liver or prostate), performing tumor therapies (e. g. by means of radio frequency ablation) or electrophysiological (EP) studies and therapies of the heart $[1,2]$.

Several of the above mentioned applications require the patient's electrocardiogram (ECG). There are two main purposes for the ECG in MRI: 1) gating of the (cardiac) MRI sequences and 2) patient monitoring.

The MRI with its different magnetic fields (strong static magnetic field, switch gradient magnetic fields in the $\mathrm{Hz}$ or $\mathrm{kHz}$ range as well as high frequency magnetic fields in the $\mathrm{MHz}$ range) makes it a challenging task to obtain a useful and reliable ECG signal during MRI. Specialized hardware as well as dedicated signal processing algorithms are required to suppress various artifacts originating from the different magnetic fields [3].

The following three subsections give a brief overview of the applications of an ECG during MRI, i. e. gating and patient monitoring, as well as its distortion caused by the so called magnetohydrodynamic (MHD) effect.

\subsection{ECG-based gating in MRI}

Gating is a technique which is used to synchronize the acquisition of the MRI raw data with the patient motion, usually respiratory or cardiac motion. One special application is cardiac MRI (CMR) where MR images of the heart are taken. Due to the rapid motion of the heart (e. g. one beat per second) and the relatively long time required to obtain a complete MR image (usually several minutes), both processes need to be synchronized. Figure 1) depicts the idea behind the ECG-based gating principle. Gating is the most important application for the ECG during MRI [4]. It can be achieved in a prospective or retrospective manner [4,5]. Other, non-ECG based methods for synchronisation make use of Doppler ultrasound, finger plethysmography, self gating or phonocardiogram techniques [6-8].

ECG-based gating requires the detection of the ECG's QRS complexes or R-peaks. Since the raw MRI data (kspace data) is collected over several cardiac cycles to reconstruct a complete MR image, data should be acquired from normal rhythm heart beats only and in a certain phase of the certain cycle [5,9]. Hence, changes in the heart rhythm such as ectopic beats should be detected in order to pause data acquisition in those periods. 


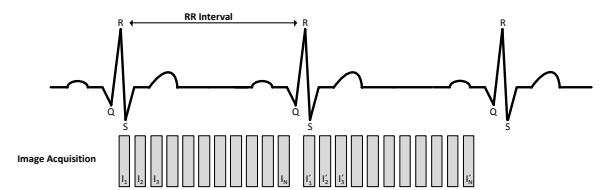

Figure 1: Principle of ECG-based gating for the acquisition of cardiac MRI data. The MRI raw data is acquired of several cardiac cycles to finally compute one static cardiac image or an animated scene of one cardiac cycle.

\subsection{ECG-based patient monitoring in MRI}

The ECG is of high importance for cardiovascular monitoring and is often used in clinical routine. Since it is often difficult to visually observe or communicate with the patient inside the MRI scanner, the physician or clinical staff has to rely on the patient's vital parameters . Especially patients who are in a critical or unstable condition (e. g. patients from an intensive care unit or patients under anesthesia), need to be adequately monitored during MRI [10]. For MRI-guided EP procedures, which are rarely performed nowadays but can become more important in the future, a diagnostically useful surface ECG is inevitable $[2,11]$.

\subsection{Distortions of the ECG during MRI}

While measuring a patient's ECG inside the MRI scanner, the various magnetic fields produce artifacts in the ECG signals resulting in a relevant loss of signal quality. Considering QRS detection and its related problems, the most severe artifacts are caused by the MRI's switched gradient magnetic fields. They induce currents inside the tissue or directly couple into the wire of the ECG device [12]. Using appropriate filtering methods, gradient artifacts can be suppressed or removed in most cases with only minor changes of the underlying ECG signal. Although far outside the ECG's frequency range, the MRI's high frequency magnetic fields (MHz range) can be demodulated by the ECG hardware resulting in additional artifacts. Both artifacts mentioned above only occur during image acquisition.

In addition to the effect of the MRI's time varying magnetic fields, the high static magnetic field of the MRI scanner affects the acquired ECG signals. The magnetohydrodynamic (MHD) effect results from the interaction between the pulsatile blood flow - which is caused by the rhythmic action of the heart - and the static magnetic field of the MR scanner, $\mathbf{B}_{0}$. These ions contained in the blood are moving inside the vessels where they experience a force due to the presence of the MR scanner's static magnetic field. This force is known as Lorentz force $\vec{F}$ :

$$
\vec{F} \propto\left(\vec{v} \times \vec{B}_{0}\right)
$$

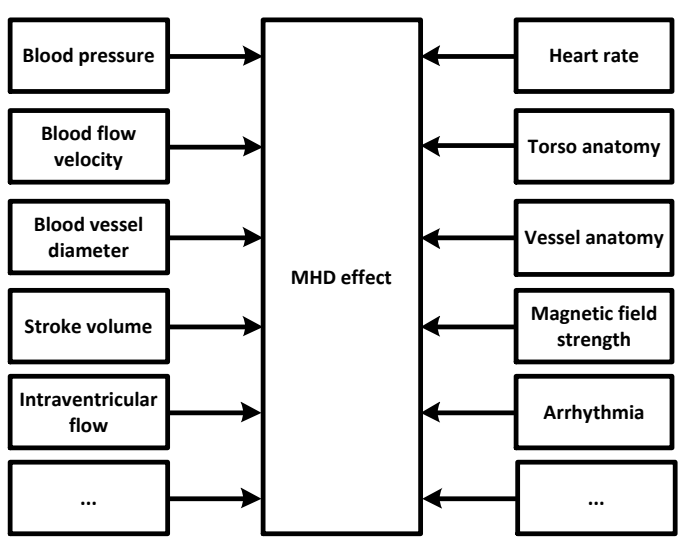

Figure 2: Selection of physiological and technical parameters affecting the MHD effect.

and depends on the magnitude and orientation of the blood flow velocity $\vec{v}$ with respect to the $\vec{B}_{0}$ field. This force causes the ions to move perpendicular to the direction of the blood flow and perpendicular to the MR scanner's static magnetic field. The ions accumulate near the vessel's wall leading to a potential difference across the vessel that may be expressed as:

$$
V \propto \int_{0}^{l} \vec{v} \times \vec{B}_{0} d \vec{l}
$$

where $l$ is the diameter of the vessel. This is the so called MHD effect. The resulting body surface potentials of the MHD effect superimpose the ECG signal. Figure 2 summarizes several physiological and technical parameters which influence the MHD effect.

When the ECG is measured inside an MR scanner, the MHD signal mainly affects the segment between two QRS complexes, i.e. the ST-segment, the T-wave and the P-wave. Exemplary ECG signals from two different subjects acquired outside and inside a $3 \mathrm{~T}$ MRI scanner are shown in Fig. 3. The diagnostic information contained in the ECG's ST-segment and T-wave is hidden by the MHD effect which reduces the diagnostic value of the ECG during an MRI scan. For very high magnetic field strengths $(\geq 7 \mathrm{~T})$, the large magnitude and slope of the MHD effect can also hamper the QRS detection [13].

\subsection{Aim of this database}

Considering the two main applications of the ECG in MRI described in Sections 1.1 and 1.2, this database aims to encourage the research community to develop algorithms which either enhance the QRS detection in ECG signals acquired at very high magnetic field strengths $(\geq 7 \mathrm{~T})$ or to improve the diagnostic value of the ECG signal. Besides this, the MHD effect could not only be considered as 


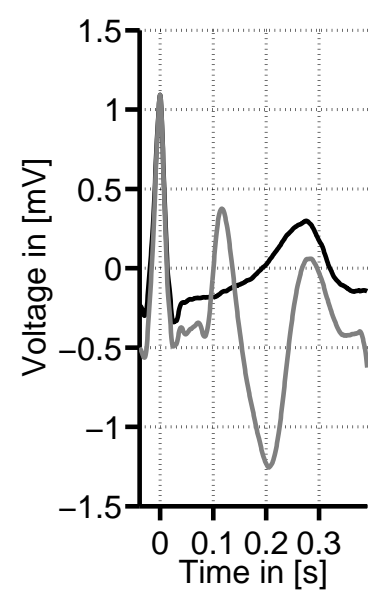

(a) Subject $\mathrm{A}$

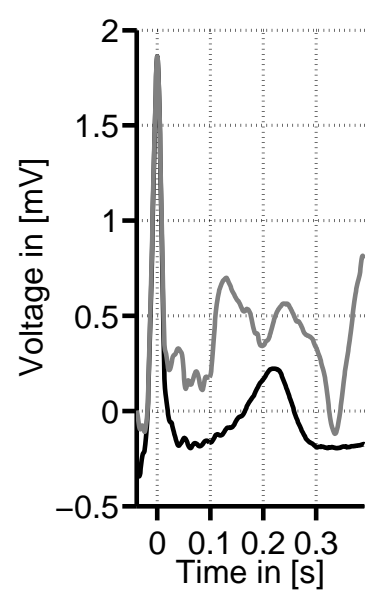

(b) Subject B
Figure 3: Comparison of the ECG signal waveform in lead II in two different subjects acquired outside (black) and inside (grey) a $3 \mathrm{~T}$ MRI scanner. The QRS complexes are aligned at $t=0 \mathrm{~s}$. The MHD effect mainly affect the ECG's ST-segmentand T-wave.

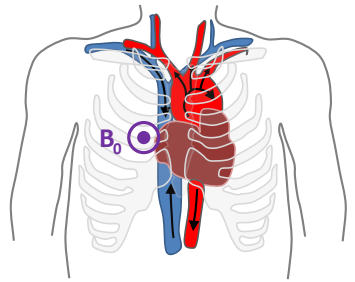

(a) Vertical $\mathrm{B}_{0}$ field (from anterior to posterior)

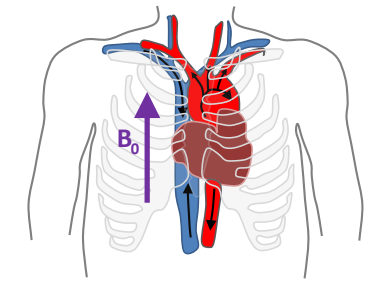

(b) Horizontal $\mathrm{B}_{0}$ field (from feet to head)
Figure 4: Alignement of the magnetic fields for a) the $1 \mathrm{~T}$ scanner and $\mathrm{b}$ ) the $3 \mathrm{~T}$ and the $7 \mathrm{~T}$ scanner.

a distortion of the ECG but as signal containing additional diagnostic information, e.g. about the blood flow velocity profile, the direction of the blood flow (aortic arch) or others.

\section{Experimental setup and subjects}

ECGs were acquired in different MRI scanners with stattic magnetic field strengths of $1 \mathrm{~T}, 3 \mathrm{~T}$ and $7 \mathrm{~T}$ without imaging, i.e. in the absence of the switched gradient and the high frequency magnetic fields. Hence, the ECG signals are only distorted by the MHD effect. For the acquisition of the ECG signals, two different ECG recorders were used: 1) a 12-lead Holter ECG (CardioMem 3000, Getemed AG, Germany) with a sampling rate of $1024 \mathrm{~Hz}$, an input voltage range of $\pm 6 \mathrm{mV}$, a resolution of 12 bit

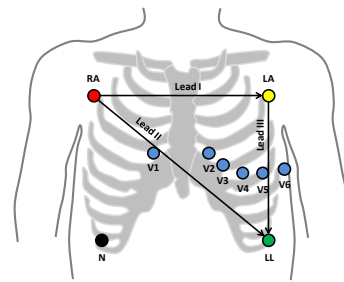

(a) 12-lead ECG

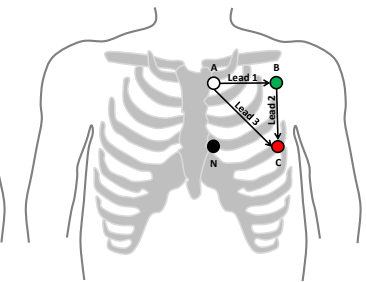

(b) 3-lead ECG ("reduced Einthoven")
Figure 5: The ECG signals of the database were either acquired using a 12-lead (a) or a 3-lead configuration (b).

Table 1: Overview of the ECG datasets included in the database.

\begin{tabular}{crrrr}
\hline Field strength & \multicolumn{2}{c}{ 12-lead ECG } & \multicolumn{2}{c}{-lead ECG } \\
& $\mathrm{N}$ & $\mathrm{L}$ & $\mathrm{N}$ & $\mathrm{L}$ \\
$1 \mathrm{~T}$ & 2 & $1 \mathrm{~min}$ & 0 & 0 \\
$3 \mathrm{~T}$ & 17 & $118 \mathrm{~min}$ & 14 & $37 \mathrm{~min}$ \\
$7 \mathrm{~T}$ & 10 & $47 \mathrm{~min}$ & 0 & 0 \\
\hline
\end{tabular}

$\mathrm{N}$ : number of records, L: overall length of the records

and an analogue bandwidth of $0.05 \mathrm{~Hz}$ to $100 \mathrm{~Hz}$ and 2) the wireless ECG device of an MRI-conditional patient monitoring system (Tesla M3, MIPM GmbH, Germany) with a sampling rate of $16 \mathrm{kHz}$ (down sampled to $1024 \mathrm{~Hz}$ ), an input voltage range of $\pm 2.4 \mathrm{mV}$ and a resolution of 24 bit. Figure 5 depicts the lead systems. The signals included in the database were acquired in the years 2012 to 2016. For its initial publication, the database comprises 43 records from 23 different subjects with an overall length of $203 \mathrm{~min}$. The subjects had an average age of $27.1 \pm 3.2$ years, an average weight of $73.8 \pm 13.1 \mathrm{~kg}$ and a height of $181.7 \pm 10.5 \mathrm{~cm}$. An overview of the ECG records is given in Table 1. The QRS complexes were manually annotated by physicians or ECG experts. No distinction was made between QRS complexes occurring in normal or a disturbed heart rhythm, e.g. caused by ectopic beats.

Most of the datasets were acquired during spontaneous, free breathing. For certain datasets, breath-hold commands were applied asking the subjects to hold their breath for several seconds in order to avoid or reduce respiratory modulations of the ECG and MHD signals.

The header file of each record contains the following information: technical parameters of the MR scanner (field strength, manufacturer, field orientation), ECG hardware parameters and information about the subject (sex, age, weight, height, position in the scanner, type of respiration). 


\section{Discussion and outlook}

Obtaining a diagnostically useful ECG signal during an MRI exam is hampered by the MRI's different magnetic fields affecting the ECG signal. The main problem one faces during the development of dedicated filters is that the underlying ECG signal is not known while the subjects are in the MRI scanner, i.e. a reference signal (ECG without distortions) does not exist. However, since all the ECG signals were acquired from healthy subjects, the ECG signals acquired outside the MRI scanner can be regarded as reference signals assuming that there are only minor morphological differences between the ECGs acquired outside and inside the MRI scanner. Hence, the signals of this database can be considered as a basis for developing filtering algorithms for ECG signals obtained during MRI scans.

The currently published database only contains ECG signal distorted by the MHD effect. This effect highly degrades the diagnostic of the ECG during MRI. We aim to extended the currently published database with additional ECG records in the future which also contain distortions caused by the MRI's switched gradient magnetic fields.

\section{Author contributions}

JWK and MS designed the experiments, acquired the ECG signals and converted them into Phyisonet's WFDB format; JWK conceived, designed and wrote the paper; GR and MF revised the final version of the paper. All authors read and approved the final version of the manuscript.

\section{Acknowledgements}

The following research grants allowed JWK and MS the acquisition of the ECG datasets: German BMBF grants 03IP710, 03IPT7100X, 03FO16101A and the German BMWi grant KF3172301JL3. The ECG recording hardware and software was provided by MIPM GmbH, Germany and by Getemed AG, Germany. PhysioNet is supported by the National Institute of General Medical Sciences (NIGMS) and the National Institute of Biomedical Imaging and Bioengineering (NIBIB) under NIH grant number 2R01GM104987-09.

\section{References}

[1] Piorkowski C, Grothoff M, Gaspar T, Eitel C, Sommer P, Huo Y, John S, Gutberlet M, Hindricks G. Cavotricuspid isthmus ablation guided by real-time magnetic resonance imaging. Circulation Arrhythmia and Electrophysiology 2013;6(1):e7-e10.

[2] Sommer P, Grothoff M, Eitel C, Gaspar T, Piorkowski C, Gutberlet M, Hindricks G. Feasibility of real-time magnetic resonance imaging-guided electrophysiology studies in humans. Europace 2013;15(1):101-108.
[3] Oster J, Clifford G. Acquisition of electrocardiogram signals during magnetic resonance imaging. Physiol Meas 2017;38(7):R119-R142.

[4] Scott A, Keegan J, Firmin D. Motion in cardiovascular MR imaging. Radiology 2009;250(2):331-351.

[5] Nacif M, Zavodni A, Kawel N, Choi E, Lima J, Bluemke D. Cardiac magnetic resonance imaging and its electrocardiographs (ECG): tips and tricks. Int J Cardiovasc Imaging 2012;28(6):1465-1475.

[6] Rubin J, Brian Fowlkes J, Prince M, Rhee R, Chenevert T. Doppler US gating of cardiac MR imaging. Acad Radiol 2000;7(12):1116-1122.

[7] Nijm G, Sahakian A, Swiryn S, Carr J, Sheehan J, Larson A. Comparison of self-gated cine MRI retrospective cardiac synchronization algorithms. J Magn Reson Imaging 2008; 28(3):767-772.

[8] Frauenrath T, Hezel F, Renz W, de Geyer d'Orth T, Dieringer M, von Knobelsdorff-Brenkenhoff F, Prothmann M, Schulz-Menger J, Niendorf T. Acoustic cardiac triggering: a practical solution for synchronization and gating of cardiovascular magnetic resonance at 7 Tesla. J Cardiovasc Magn Reson 2010;12(67):1-14.

[9] Fernandez B, Oster J, Lohézic M, Mandry D, Pietquin O, Vuissoz P, Felblinger J. Adaptive Black Blood Fast Spin Echo for End-Systolic Rest Cardiac Imaging. Magnet Reson Med 2010;64(6):1760-1771.

[10] American Society of Anesthesiologists: Standards for Basic Anesthetic Monitoring, 2011.

[11] Razavi R, Mutharangu V, Hegde S, Taylor A. Clinical Cardiac MRI. Springer, 2004; 513-538.

[12] Felblinger J, Slotboom J, Kreis R, Jung B, Boesch C, et al. Restoration of Electrophysiological Signals Distorted by Inductive Effects of Magnetic Field Gradients During MR Sequences. Magnet Reson Med 1999;41(4):715-721.

[13] Krug J, Rose G, Clifford G, Oster J. ECG-Based Gating in Ultra High Field Cardiac MRI using an Independent Component Analysis Approach. J Cardiovasc Magn Reson 2013;15(104):1-13.

Address for correspondence:

Johannes Krug // krug.johannes at gmail.com Otto-von-Guericke-University Magdeburg Universitaetsplatz 2 // 39106 Magdeburg // Germany 